\title{
The use of telemedicine in the management of acute stroke
}

\author{
Mark N. Rubin, M.D., ${ }^{1}$ AND Bart M. Demaerschalk, M.D., M.Sc., F.R.C.P.C. ${ }^{2}$ \\ ${ }^{\prime}$ Department of Neurology, Mayo Clinic, Jacksonville, Florida; and ${ }^{2}$ Department of Neurology, Mayo Clinic, \\ Phoenix, Arizona
}

\begin{abstract}
Cerebrovascular disease, including acute ischemic stroke, remains a major public health problem in the US and throughout the world. There has been a concerted effort to apply evidence-based practices to stroke care to improve primary and secondary prevention as well as poststroke outcomes. Geography and workforce shortages contribute to a disparity in stroke care, however, among the substantial proportion of the US population that lives outside the reach of an acute stroke-ready hospital or a primary or comprehensive stroke center. In an attempt to combat the rural-to-urban disparity and expand the availability of best stroke practices, Levine and Gorman proposed the development of telemedical outreach for acute stroke evaluation and management, which they called "telestroke." Since then, the practice of telestroke has been found to have a high interrater agreement with a bedside assessment of the National Institutes of Health Stroke Scale score, to enhance correct thrombolysis decision making as compared with telephone-only consultation, and to be cost-effective. In light of these findings and the perception of benefit by acute stroke providers and patients, there has been growing interest in and a rapid expansion of telestroke networks in the US and internationally. There are legal and financial barriers to more widespread use of telemedicine in general, including telestroke. Further research is needed to understand the potential merits of telestroke infrastructure for the many phases of stroke care including poststroke hospitalization, prevention of complications, enhancing secondary prevention, and education of patients and providers.

(http://thejns.org/doi/abs/10.3171/2013.11.FOCUS13428)
\end{abstract}

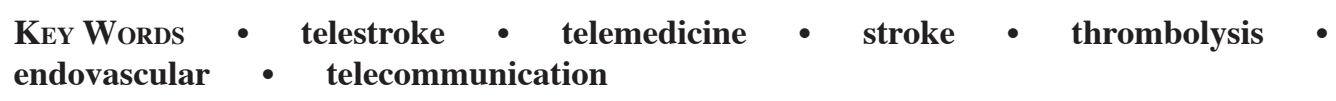

$\mathrm{C}$ EREBROVASCULAR disease, including acute ischemic stroke, remains a major public health problem in the US $^{33}$ and throughout the world. ${ }^{5}$ There has been a concerted effort to apply evidence-based practices to stroke care to improve primary and secondary prevention as well as poststroke outcomes. One facet of this effort includes the development and accreditation of acute stroke-ready hospitals, and primary and comprehensive stroke centers, which have been demonstrated to improve stroke care. ${ }^{21,27}$ The prompt and guideline-based administration of an acute stroke evaluation and management are among the more heavily scrutinized aspects of a stroke center. ${ }^{14}$ The rationale for the particular attention to expedient administration of acute stroke evaluation and therapy is sound, given the limited time window for administration of the only FDA-approved therapy for acute stroke, recombinant tissue plasminogen activator (rt-PA)..$^{19,31}$

Geography contributes to a disparity in stroke care, however, because most stroke centers are based in large urban academic medical centers. It is estimated that upwards of $40 \%$ of the US population resides outside the reasonable clinical reach of a primary stroke center. This previously presented a considerable barrier to the timely

\footnotetext{
Abbreviations used in this paper: ICER = incremental cost-effectiveness ratio; NIHSS = National Institutes of Health Stroke Scale; QALY = quality-adjusted life year; rt-PA = recombinant tissue plasminogen activator; $4 \mathrm{G}=$ fourth-generation (mobile) network.
}

administration of acute stroke therapy. Furthermore, there remains a shortage of vascular neurologists, who are otherwise best equipped to provide desired outcomes. . $^{10,17,42}$

In an attempt to combat the rural-to-urban disparity and expand the availability of best stroke practices, Levine and Gorman proposed the development of telemedical outreach for acute stroke evaluation and management, which they called "telestroke." 26 Since then, the practice of telestroke has been found to have a high interrater agreement with a bedside assessment of the National Institutes of Health Stroke Scale (NIHSS) score, , ,39,45 to enhance correct rt-PA decision making as compared with telephone-only consultation,,$^{6,8,29}$ and to be cost-effective. ${ }^{32,44}$ In light of these findings and the perception of benefit by acute stroke providers and patients, there has been growing interest in and a rapid expansion of telestroke networks both in the US ${ }^{16,40}$ and internationally. There has been less material published on and seemingly less use of telestroke infrastructure for the complex, multifaceted, and similarly important subacute phase of stroke evaluation and management. ${ }^{36}$

\section{Acute Ischemic Stroke}

\section{Evidence for Telestroke}

Most telestroke manuscripts as of 2012 have focused on the acute phase of stroke care (155 for acute stroke, 28 for poststroke) ${ }^{35,36}$ The state of telestroke practice has matured to the point that there are specific American 


\section{N. Rubin and B. M. Demaerschalk}

Heart Association/American Stroke Association statements detailing the evidence for its use ${ }^{38}$ and guidelines for implementation..$^{22,37}$ This came to be on the strength of studies that suggest excellent interrater reliability of NIHSS examination between remote and bedside examiners, ${ }^{22,40,46}$ randomized controlled trials of telestroke versus telephone consultation for acute stroke that demonstrate superiority of telestroke for thrombolysis decision-making, ${ }^{6,29}$ and favorable health economic analyses (see below). ${ }^{32,44}$ Telestroke is now considered to be in the mainstream of clinical practice in the academic and community environments..$^{16,40}$ Furthermore, it has been demonstrated that high-quality telestroke consultations can be performed with mobile computers ${ }^{3}$ and smartphones, ${ }^{1,9,18}$ enhancing the portability of the service.

There remain only two published randomized controlled trials of telestroke versus telephone methods of consultation for consideration of thrombolysis of acute ischemic stroke as of late 2013. The first trial, published in 2008, randomized 222 patients (111 for each study arm) with acute ischemic stroke to either telephone-only or telestroke-guided evaluation. The primary outcome was adjudication of "correct treatment" with rt-PA by National Institute of Neurological Disorders and Stroke criteria. Typical stroke metrics were also tracked. In brief, the rt-PA decision was adjudicated to be correct significantly more often with telestroke $(98 \%)$ than telephone $(82 \%)$ consultations. In spite of the telephone group having a significantly lower NIHSS score on presentation (7.7) as compared with the telestroke group (11.4), there were no differences in 90-day mortality or outcome, nor were there any differences in rate of hemorrhage..$^{29} \mathrm{~A}$ second group emulated this methodology with the intent of demonstrating the feasibility of a trial of telestroke versus telephone consultation for acute ischemic stroke in another region (for example, underpowered to demonstrate superiority of one mode over another). Fifty-four patients participated (27 for each arm) and no consultations were aborted, but technical issues were frequent in the telestroke arm. Adjudicated rt-PA decision making was similar and good between the telephone (89\%) and telestroke (85\%) groups. There were no differences in 90 day mortality or outcome, nor were there any differences in rate of hemorrhage. ${ }^{6} \mathrm{~A}$ pooled analysis of these identically designed trials supports the conclusions of the original trial, with a correct rt-PA decision significantly more likely with telestroke (96\%) versus telephone (83\%), with excellent frequency of rt-PA use (26\%) and no difference in mortality rates, outcomes, or hemorrhage. ${ }^{8}$

There are a substantial number of pilot and feasibility studies that make up the foundation of the field, detailing how to incorporate telestroke effectively into practice. The field appears to be advancing, but primarily via creative postimplementation studies, particularly in the prehospital setting. A group of investigators at the University of Maryland, otherwise known by the moniker "TeleBAT," published the early data on prehospital telestroke, ${ }^{24,25}$ and although they demonstrated reasonable interrater agreement of NIHSS scores between onsite and telestroke providers, their reported technology is antiquated. A German group reported on their pilot, ${ }^{11}$ called "PHANTOM-S," in which enhanced stroke-dedicated ambulances equipped with CT scanners, point of care laboratory, teleradiology, and telestroke capabilities were used. However, their initial experience yielded an unacceptably high rate of technical failures. ${ }^{28} \mathrm{~A}$ subsequent study, however, using a modern fourth-generation (4G) mobile network for data transmission, demonstrated feasibility and excellent call-to-needle times for treated patients. ${ }^{46}$ Further study is planned, and the potential benefit to individual patients as well as society is tremendous.

\section{Intracranial Hemorrhage}

Telestroke for acute intracranial hemorrhage-subdural, subarachnoid, or intraparenchymal-is not as well studied as its use for acute ischemic stroke but, as a group, these disorders represent a substantial minority of indications for a telestroke consultation. ${ }^{23}$ The use of telestroke technology for early identification of candidates for emergency hemicraniectomy and/or hematoma evacuation has been studied sparingly.,12 A single-center retrospective review of an experience with a hub-and-spoke model of teleconsultation for intracerebral hemorrhage suggests that the use of telemedicine for this indication is feasible. The nature of the study does not allow for inference of clear benefit for patients seen by telemedicine versus usual care, but the potential benefits of extension of neurosurgical expertise is perhaps self-evident. An interesting recent pilot study of remote proctoring of general surgery residents by specialist surgeons was conducted, and one of the tested procedures was hemicraniectomy and subdural hematoma evacuation. Overall, resident operative performance was deemed significantly better with remote proctoring by a specialty surgeon as compared with an unsupervised attempt. There have been no such pilots for teleproctoring of these neurosurgical procedures on patients with intracranial hemorrhage to date.

\section{Poststroke Evaluation and Care}

Of the 18 studies to date that contribute primary data on the use of telestroke technology for poststroke evaluation and care, all represented small pilots and were exploratory in nature. There were no randomized controlled trials, economic analyses, or postimplementation studies. Of note, nearly one-third of the manuscripts (10 of 28) were narrative reviews and opinion pieces. ${ }^{36}$

Many of the published manuscripts for poststroke telestroke come from the physiatry literature and detail pilot studies of home telerehabilitation systems for patients who have experienced a stroke. The studies that evaluated videoconferencing infrastructure for other, nonphysiatric elements of poststroke care are limited but promising. For example, an interesting pilot study conducted by Mikulik et al. ${ }^{30}$ compared the logistics of performing a transcranial Doppler and carotid duplex examination by telemedical guidance of a novice versus an in-person examination by an experienced sonographer. They performed telemedical and in-person studies in each of 8 patients. There was reasonable agreement in the findings, particularly in the $7 \mathrm{pa}-$ tients with sonographically normal carotid and intracranial vasculature. These investigators concluded that telemedical guidance of transcranial Doppler and carotid duplex 
studies by an experienced sonographer was feasible for nonurgent studies and had good agreement with in-person studies in patients with normal vasculature.

Another aspect of poststroke care for patients with aphasia is a consultation with a speech pathologist. A pilot study by Brennan et al. ${ }^{4}$ sought to determine if telemedicine is an effective means of providing this service. They studied 40 patients who each underwent in-person and telemedical observation while performing the Story Retelling Procedure. The goal was to identify any difference in performance between the experimental (that is, telemedical) and control (that is, in-person) settings and, if any were found, to associate them with any demographic features such as age, sex, or experience with technology. No significant differences were found in performance between the two settings, and no demographic features predicted particularly good or poor performances in any setting. The telemedical method was also highly satisfactory to participants. The authors concluded that videoconferencing has potential in poststroke aphasia evaluation, but requires more investigation.

\section{Health Economic Analysis}

Telestroke practice is at a stage where health economic analyses have been performed and suggest societal costeffectiveness $^{32}$ and long-term cost savings from the hospital perspective. ${ }^{44}$ The first telestroke economic analysis was designed to estimate the societal cost of telestroke for delivery of acute stroke therapy at 90-day and lifetime horizons versus usual care. A decision-analytical model was used and data inputs came from the clinical experience of the investigators, assuming a network of a single receiving ("hub") and 8 referring ("spoke") centers. Costs and outcome estimations were based on studies current as of 2008. Briefly, it was shown that telestroke for delivery of thrombolysis is more cost-effective in the lifetime horizon as compared with usual care, with an incremental cost-effectiveness ratio (ICER) of \$2449 per quality-adjusted life year (QALY), more so than in the 90-day horizon (ICER of $\$ 108,363 / \mathrm{QALY}$ ). The authors suggested that the divergence of results by time horizon is most likely due to the large upfront fixed costs of telestroke equipment compared with the lifelong benefit of better neurological outcomes. ${ }^{32}$

Following that study, others sought to model the costrelated aspects of stroke care for spoke and hub institutions more specifically with and without a telestroke network in place. They used a decision-analytical model and shaped the "with telestroke network" and "no telestroke network" on their considerable clinical experience with referring centers. Costs and outcome estimations were based on studies current as of 2011. The analysis assumed a single hub and a 7-spoke network. In brief, with the telestroke network in place, the model predicted that approximately 114 fewer stroke patients would be admitted to the hub hospital each year, whereas approximately 16 more patients would be admitted to each spoke hospital compared with a no-network setting. The model predicted that approximately 45 more patients would be expected to be treated with intravenous thrombolysis and 20 more with endovascular stroke therapy in a telestroke network per year. From the entire network perspective, an estimated average cost savings of $\$ 358,435$ per year could be achieved with a telestroke network versus a network without telestroke during the first 5 years. The hub would bear positive costs of $\$ 405,121$ per year, but each spoke would save $\$ 109,080$ per year. With cost-sharing arrangements between the hub and spoke hospitals, this analysis suggests that each hospital could achieve equal cost savings of $\$ 44,804$ per year during a 5-year time horizon. Overall, the results of this study suggest that a telestroke network may be an effective and financially tenable way to extend the reach of stroke specialists to remote areas and thus to improve the overall quality of care for stroke patients. ${ }^{44}$

\section{Telestroke Technology}

The term "telestroke" has been defined as "live, audiovideo telecommunication applied to care of acute stroke."7 Historically, telestroke was practiced by a number of technological means less sophisticated than videoconferencing, including telephone, ${ }^{15}$ Multimedia Messaging Service, ${ }^{41}$ email, or some combination thereof. Although no evidence-based technological standards exist for telestroke, most modern telestroke systems are based on high-quality videoconferencing, which an American Heart Association/ American Stroke Association guideline defines as a system that "... includ[es] transmission rates and algorithms of sufficient quality to support $>20$ frames per second of bidirectional synchronized audio and video at a resolution capable of being accurately displayed on monitors of $\geq 13$ in." 38 These represent minimum standards, however, and are not strictly evidence-based. Furthermore, it seems that each of the many telestroke networks that now exist have different technology in place to fit their idiosyncratic practice needs. The technological aspects of a telestroke network are of interest because there has been growth in the telestroke-related telecommunications market within the last decade, and the cost thereof remains one of the most consistently identified barriers to implementation of a telestroke network. ${ }^{13,43}$ Furthermore, in addition to hardware specifications, the desire for mobile telestroke capability (for example, prehospital telestroke) requires technical and privacy standards for wireless data transmission.

\section{Legal and Legislative Issues}

In spite of a robust and growing evidence base supporting the use of telemedicine in general and telestroke in particular, there are a host of legal considerations that constitute a barrier to more widespread implementation. Among them are disparate licensing and credentialing requirements between each state or nation.

In the US, a physician must be licensed in the state in which a patient seeks care. Thus, a telemedicine physician must be licensed in each and every state involved in the telemedicine network. A recent piece of federal legislation (42 CFR $\$ \S 482.12$ and 482.22) helped to streamline the process of being credentialed for a telemedicine site by allowing the credentialing process of the hub site effectively to "transfer" so as to avoid duplicative administrative barriers.

Many of the legal and legislative issues exist for the use of telemedicine in general, but there are some that 
are particularly relevant to telestroke. Some who are wary of developing a telestroke network cite the lack of legal clarity at a federal level (or even in most states) regarding shared liability between hub and spoke sites in the case of a bad outcome. For the case of acute stroke, because it seems that the majority of stroke-related lawsuits come from rt-PA not being administered, institution of a process that affords emergency medicine providers access to stroke specialists and has been shown to increase rt-PA use should mitigate this concern. That said, there is still a role for establishing clear legal agreements between hub and spoke sites, be they via federal law or on an individual basis.

\section{Considerations}

Telemedicine allows a provider to extend expertise far and wide, skirting geographical barriers, and has the potential to facilitate more timely, efficient, and effective care to all, irrespective of medical specialty. The physical and logistical considerations of the general practitioner making house calls via horse-drawn carriage seem truly antiquated in the era of $4 \mathrm{G}$ wireless infrastructure; however, some basic principles of provider-patient interaction that we as providers have espoused since those house-call days remain entirely relevant to telemedical practice. More specifically, we as physicians cannot allow anything-telemedicine or otherwise-to jeopardize the ineffable healing power of an empathy-driven interaction with a patient. Ostensibly, an inability to touch a shoulder or overtly display sympathetic body language to the patient and their loved ones might disallow the nontechnical aspects of a medical consultation. That said, a telemedical consultation can be conducted in such a fashion as to maximize empathic interaction, with language and deliberate physical gestures to make up for an inability to tacitly convey our sympathies as we otherwise might in person. In other words, the "human touch" can be achieved with telemedicine. At the time of this writing, at least one study of perceived empathy through telemedical consultation is actively enrolling, and a second study of patient and provider satisfaction with telemedicine is being designed. We hope that these studies and others will demonstrate that, with a commitment to empathic care on the part of a provider, the provider-patient relationship does not substantially differ between in-person and telemedical consultation.

\section{Future Directions}

More than a decade since its published conceptualization, there is now a robust and growing literature base that supports the use of telestroke in mainstream clinical stroke practice. It is noteworthy that telemedicine publications in acute stroke represent approximately $40 \%$ of all published articles on telemedicine applied to the broad field of clinical neurological sciences and all of its subspecialties. ${ }^{34}$ The trajectory of telestroke research is mostly encouraging given the recent flurry of postimplementation studies, particularly in the prehospital setting, which aim to further reduce time to stroke recognition and treatment. Of particular interest is the role of prehospital telestroke for diagnosis of a large-artery stroke syndrome in patients who might benefit from early triage to endovascular reperfusion therapy.

Further study is recommended to establish minimum technical standards for in- and prehospital telestroke use. The use of telestroke videoconferencing infrastructure for education of trainees and the community at large about acute stroke evaluation and management also remains largely unstudied. Perhaps most importantly, there is a paucity of randomized trials and cost analyses, which might otherwise serve to buttress the practice and dissolve barriers to the implementation of telestroke. Overall, however, telestroke practice and its evidence base continue to grow, to the benefit of stroke patients.

\section{Disclosure}

The authors report no conflict of interest concerning the materials or methods used in this study or the findings specified in this paper.

Author contributions to the study and manuscript preparation include the following. Conception and design: both authors. Analysis and interpretation of data: both authors. Drafting the article: both authors. Critically revising the article: Demaerschalk. Reviewed submitted version of manuscript: Demaerschalk. Approved the final version of the manuscript on behalf of both authors: Demaerschalk. Study supervision: Demaerschalk.

\section{References}

1. Anderson ER, Smith B, Ido M, Frankel M: Remote assessment of stroke using the iPhone 4. J Stroke Cerebrovasc Dis 22:340-344, 2013

2. Angileri FF, Cardali S, Conti A, Raffa G, Tomasello F: Telemedicine-assisted treatment of patients with intracerebral hemorrhage. Neurosurg Focus 32(4):E6, 2012

3. Audebert HJ, Boy S, Jankovits R, Pilz P, Klucken J, Fehm NP, et al: Is mobile teleconsulting equivalent to hospital-based telestroke services? Stroke 39:3427-3430, 2008

4. Brennan DM, Georgeadis AC, Baron CR, Barker LM: The effect of videoconference-based telerehabilitation on story retelling performance by brain-injured subjects and its implications for remote speech-language therapy. Telemed J E Health 10:147-154, 2004

5. Brundtland GH: From the World Health Organization. Reducing risks to health, promoting healthy life. JAMA 288:1974, 2002

6. Demaerschalk BM, Bobrow BJ, Raman R, Kiernan TE, Aguilar MI, Ingall TJ, et al: Stroke team remote evaluation using a digital observation camera in Arizona: the initial mayo clinic experience trial. Stroke 41:1251-1258, 2010

7. Demaerschalk BM, Miley ML, Kiernan TE, Bobrow BJ, Corday DA, Wellik KE, et al: Stroke telemedicine. Mayo Clin Proc 84:53-64, 2009

8. Demaerschalk BM, Raman R, Ernstrom K, Meyer BC: Efficacy of telemedicine for stroke: pooled analysis of the Stroke Team Remote Evaluation Using a Digital Observation Camera (STRokE DOC) and STRokE DOC Arizona telestroke trials. Telemed J E Health 18:230-237, 2012

9. Demaerschalk BM, Vegunta S, Vargas BB, Wu Q, Channer DD, Hentz JG: Reliability of real-time video smartphone for assessing National Institutes of Health Stroke Scale scores in acute stroke patients. Stroke 43:3271-3277, 2012

10. Donnan GA, Davis SM: Neurologist, internist, or strokologist? Stroke 34:2765, 2003

11. Ebinger M, Rozanski M, Waldschmidt C, Weber J, Wendt M, Winter B, et al: PHANTOM-S: the prehospital acute neurological therapy and optimization of medical care in stroke patients - study. Int J Stroke 7:348-353, 2012 
12. Ereso AQ, Garcia P, Tseng E, Gauger G, Kim H, Dua MM, et al: Live transference of surgical subspecialty skills using telerobotic proctoring to remote general surgeons. J Am Coll Surg 211:400-411, 2010

13. Fanale CV, Demaerschalk BM: Telestroke network business model strategies. J Stroke Cerebrovasc Dis 21:530-534, 2012

14. Fonarow GC, Gregory T, Driskill M, Stewart MD, Beam C, Butler J, et al: Hospital certification for optimizing cardiovascular disease and stroke quality of care and outcomes. Circulation 122:2459-2469, 2010

15. Frey JL, Jahnke HK, Goslar PW, Partovi S, Flaster MS: tPA by telephone: extending the benefits of a comprehensive stroke center. Neurology 64:154-156, 2005

16. George BP, Scoglio NJ, Reminick JI, Rajan B, Beck CA, Seidmann A, et al: Telemedicine in leading US neurology departments. Neurohospitalist 2:123-128, 2012

17. Goldstein LB, Matchar DB, Hoff-Lindquist J, Samsa GP, Horner RD: VA Stroke Study: neurologist care is associated with increased testing but improved outcomes. Neurology 61:792-796, 2003

18. Gonzalez MA, Hanna N, Rodrigo ME, Satler LF, Waksman $\mathrm{R}$ : Reliability of prehospital real-time cellular video phone in assessing the simplified National Institutes of Health Stroke Scale in patients with acute stroke: a novel telemedicine technology. Stroke 42:1522-1527, 2011

19. Hacke W, Kaste M, Bluhmki E, Brozman M, Dávalos A, Guidetti D, et al: Thrombolysis with alteplase 3 to 4.5 hours after acute ischemic stroke. N Engl J Med 359:1317-1329, 2008

20. Handschu R, Littmann R, Reulbach U, Gaul C, Heckmann JG, Neundörfer B, et al: Telemedicine in emergency evaluation of acute stroke: interrater agreement in remote video examination with a novel multimedia system. Stroke 34:2842-2846, 2003

21. Higashida R, Alberts MJ, Alexander DN, Crocco TJ, Demaerschalk BM, Derdeyn CP, et al: Interactions within stroke systems of care: a policy statement from the American Heart Association/American Stroke Association. Stroke 44:29612984, 2013

22. Jauch EC, Saver JL, Adams HP Jr, Bruno A, Connors JJ, Demaerschalk BM, et al: Guidelines for the early management of patients with acute ischemic stroke: a guideline for healthcare professionals from the American Heart Association/American Stroke Association. Stroke 44:870-947, 2013

23. Kepplinger J, Dzialowski I, Barlinn K, Puetz V, Wojciechowski C, Schneider H, et al: Emergency transfer of acute stroke patients within the East Saxony telemedicine stroke network: a descriptive analysis. Int J Stroke [epub ahead of print], 2013

24. LaMonte MP, Cullen J, Gagliano DM, Gunawardane R, Hu P, Mackenzie C, et al: TeleBAT: mobile telemedicine for the Brain Attack Team. J Stroke Cerebrovasc Dis 9:128-135, 2000

25. LaMonte MP, Xiao Y, Hu PF, Gagliano DM, Bahouth MN, Gunawardane RD, et al: Shortening time to stroke treatment using ambulance telemedicine: TeleBAT. J Stroke Cerebrovasc Dis 13:148-154, 2004

26. Levine SR, Gorman M: "Telestroke": the application of telemedicine for stroke. Stroke 30:464-469, 1999

27. Lichtman JH, Allen NB, Wang Y, Watanabe E, Jones SB, Goldstein LB: Stroke patient outcomes in US hospitals before the start of the Joint Commission Primary Stroke Center certification program. Stroke 40:3574-3579, 2009

28. Liman TG, Winter B, Waldschmidt C, Zerbe N, Hufnagl P, Audebert HJ, et al: Telestroke ambulances in prehospital stroke management: concept and pilot feasibility study. Stroke 43:2086-2090, 2012

29. Meyer BC, Raman R, Hemmen T, Obler R, Zivin JA, Rao R, et al: Efficacy of site-independent telemedicine in the STRokE DOC trial: a randomised, blinded, prospective study. Lancet Neurol 7:787-795, 2008

30. Mikulik R, Alexandrov AV, Ribo M, Garami Z, Porche NA, Fulep E, et al: Telemedicine-guided carotid and transcranial ultrasound: a pilot feasibility study. Stroke 37:229-230, 2006
31. National Institute of Neurological Disorders and Stroke rt-PA Stroke Study Group: Tissue plasminogen activator for acute ischemic stroke. N Engl J Med 333:1581-1587, 1995

32. Nelson RE, Saltzman GM, Skalabrin EJ, Demaerschalk BM, Majersik JJ: The cost-effectiveness of telestroke in the treatment of acute ischemic stroke. Neurology 77:1590-1598, 2011

33. Roger VL, Go AS, Lloyd-Jones DM, Benjamin EJ, Berry JD, Borden WB, et al: Heart disease and stroke statistics-2012 update: a report from the American Heart Association. Circulation 125:e2-e220, 2012

34. Rubin MN, Wellik KE, Channer DD, Demaerschalk BM: Role of telemedicine in providing tertiary neurological care. Curr Treat Options Neurol 15:567-582, 2013

35. Rubin MN, Wellik KE, Channer DD, Demaerschalk BM: A systematic review of telestroke. Postgrad Med 125:45-50, 2013

36. Rubin MN, Wellik KE, Channer DD, Demaerschalk BM: Systematic review of telestroke for post-stroke care and rehabilitation. Curr Atheroscler Rep 15:343, 2013

37. Schwamm LH, Audebert HJ, Amarenco P, Chumbler NR, Frankel MR, George MG, et al: Recommendations for the implementation of telemedicine within stroke systems of care: a policy statement from the American Heart Association. Stroke 40:2635-2660, 2009

38. Schwamm LH, Holloway RG, Amarenco P, Audebert HJ, Bakas T, Chumbler NR, et al: A review of the evidence for the use of telemedicine within stroke systems of care: a scientific statement from the American Heart Association/American Stroke Association. Stroke 40:2616-2634, 2009

39. Shafqat S, Kvedar JC, Guanci MM, Chang Y, Schwamm LH: Role for telemedicine in acute stroke. Feasibility and reliability of remote administration of the NIH stroke scale. Stroke 30:2141-2145, 1999

40. Silva GS, Farrell S, Shandra E, Viswanathan A, Schwamm LH: The status of telestroke in the United States: a survey of currently active stroke telemedicine programs. Stroke 43:20782085, 2012

41. Singh R, Ng WH, Lee KE, Wang E, Ng I, Lee WL: Telemedicine in emergency neurological service provision in Singapore: using technology to overcome limitations. Telemed J E Health 15:560-565, 2009

42. Smith MA, Liou JI, Frytak JR, Finch MD: 30-day survival and rehospitalization for stroke patients according to physician specialty. Cerebrovasc Dis 22:21-26, 2006

43. Switzer JA, Demaerschalk BM: Overcoming challenges to sustain a telestroke network. J Stroke Cerebrovasc Dis 21: 535-540, 2012

44. Switzer JA, Demaerschalk BM, Xie J, Fan L, Villa KF, Wu EQ: Cost-effectiveness of hub-and-spoke telestroke networks for the management of acute ischemic stroke from the hospitals' perspectives. Circ Cardiovasc Qual Outcomes 6:18-26, 2013

45. Wang S, Lee SB, Pardue C, Ramsingh D, Waller J, Gross H, et al: Remote evaluation of acute ischemic stroke: reliability of National Institutes of Health Stroke Scale via telestroke. Stroke 34:e188-e191, 2003

46. Weber JE, Ebinger M, Rozanski M, Waldschmidt C, Wendt M, Winter B, et al: Prehospital thrombolysis in acute stroke: results of the PHANTOM-S pilot study. Neurology 80:163168,2013

Manuscript submitted September 25, 2013.

Accepted November 19, 2013.

Please include this information when citing this paper: DOI: 10.3171/2013.11.FOCUS13428.

Address correspondence to: Bart M. Demaerschalk, M.D., M.Sc., F.R.C.P.C., Department of Neurology, Mayo Clinic, 5777 E. Mayo Clinic Blvd., Phoenix, AZ 85254. email: demaerschalk.bart@mayo. edu. 